\title{
Objective tumor distinction in 5-aminolevulinic acid-based endoscopic photodynamic diagnosis, using a spectrometer with a liquid crystal tunable filter
}

\author{
Taro Yamashita, Hidehito Kinoshita, Takuki Sakaguchi, Hajime Isomoto \\ Faculty of Medicine, Division of Medicine and Clinical Science, Department of Multidisciplinary Internal Medicine, Tottori University, \\ Tottori, Japan \\ Contributions: (I) Conception and design: T Yamashita; (II) Administrative support: T Yamashita; (III) Provision of study materials or patients: H \\ Kinoshita, T Sakaguchi; (IV) Collection and assembly of data: H Kinoshita, T Sakaguchi; (V) Data analysis and interpretation: T Yamashita, H \\ Isomoto; (VI) Manuscript writing: All authors; (VII) Final approval of manuscript: All authors. \\ Correspondence to: Hajime Isomoto. Faculty of Medicine, Division of Medicine and Clinical Science, Department of Multidisciplinary Internal \\ Medicine, Tottori University, Nishicho 36-1, Yonago City, Tottori Prefecture, Japan. Email: isomoto@med.tottori-u.ac.jp.
}

Background: Recent improvement of the endoscopic system such as image enhancement has led to the
better accuracy of the diagnosis of the gastric cancer. However, the objective and efficient detection method
of the gastric cancer is still needed because the detection efficiency could sometimes be low due to the
fact that the image enhancement diagnosis needs magnification for its full utilization. The photodynamic
diagnosis (PDD) with oral intake of 5-aminolevulinic acid (5-ALA) has been widely used for the detection
of the cancerous region for bladder cancer and glioblastoma. The application of the 5-ALA based PDD (5-
ALA PDD) to the diagnosis of gastric cancer is recently reported. The efficiency of the detection is reported
to be good, however, the objectivity of the method can be impaired by the photobleaching effect with fast
decreasing of the intensity of the fluorescence under light exposure. In this article, we investigated the
fluorescence spectrum of the gastric tumor and non-tumor mucosa of 5-ALA PDD and revealed the property
of the photobleaching effect.

Methods: Example cases of PDD endoscopy of gastric tumor were investigated for cases of endoscopic submucosal dissection (ESD). Newly developed spectrometer using a spectrometer with a liquid crystal tunable filter was used for investigating the fluorescence spectrum of 5-ALA PDD. The assumed tumor region and non-tumor region in gastric mucosa were biopsied and the fluorescence spectrum was measured using the spectrometer consecutively several times, to estimate the photobleaching effect.

Results: The fluorescence spectrum has a primary peak at $630 \mathrm{~nm}$, with a broad peak ranging from 660 to $700 \mathrm{~nm}$. The $630 \mathrm{~nm}$ peak diminished quickly upon ultraviolet light exposure, whereas the broad peak from 660 to $700 \mathrm{~nm}$ diminished slowly. The sum of the altitudes at $660-700 \mathrm{~nm}$, normalized to the altitude at $600 \mathrm{~nm}$, was not as affected by the photobleaching effect as the $630 \mathrm{~nm}$ peak was, and can thus be used for 5-ALA-based PDD.

Conclusions: The 5-ALA PDD using the average fluorescence altitude of 660-700 nm instead of the peak altitude at $630 \mathrm{~nm}$, is shown to be more effective in distinguishing between tumorous and non-tumorous tissues, because of the lower photobleaching effect at this specific spectral range. The finding is expected to greatly improve the objective diagnosis of gastrointestinal cancers by 5-ALA-based photodynamic diagnostic endoscopy.

Keywords: Photodynamic diagnosis (PDD); 5-aminolevulinic acid (5-ALA); photobleaching effect

Submitted Nov 09, 2019. Accepted for publication Jan 10, 2020.

doi: 10.21037/atm.2020.01.108

View this article at: http://dx.doi.org/10.21037/atm.2020.01.108 


\section{Introduction}

The fluorescence properties of gastric tumors and the gastric mucosa, following oral intake of 5 -aminolevulinic acid (5-ALA) by the patients, were investigated using a newly developed spectrometer with a liquid crystal tunable filter. The fluorescence spectrum has a primary peak at $630 \mathrm{~nm}$, with a broad peak ranging from 660 to $700 \mathrm{~nm}$. One of the sources of the spectrum is known to be protoporphyrin IX, a metabolite of 5-ALA. The intensity of fluorescent light diminishes upon exposure to ultraviolet light, which is a property known as the photobleaching effect. In this study, the photobleaching effect on the fluorescence intensity and spectrum of gastric tumors was investigated. The $630 \mathrm{~nm}$ peak diminished quickly upon ultraviolet light exposure, whereas the broad peak from 660 to $700 \mathrm{~nm}$ diminished slowly. The sum of the altitudes at $660-700 \mathrm{~nm}$, normalized to the altitude at $600 \mathrm{~nm}$, was not as affected by the photobleaching effect as the $630 \mathrm{~nm}$ peak was, and can thus be used for 5-ALA-based photodynamic diagnosis (PDD). The method was investigated and successfully tested for biopsied samples in example cases of gastric tumors, using newly developed spectrometer with liquid crystal tunable filter.

The diagnosis of gastric cancer has been progressing together with the development of the endoscopic system. Recently, an image-enhanced endoscopic system named narrow band imaging (NBI) was developed for the improved detection of blood vessels, resulting in the better diagnosis of gastric cancers (1-3). In stomach, multiple tumor regions frequently exist, since background mucosa has high cancer risk due to the inflammation with the infection to Helicobacter pylori. Additionally, it is reported that the accurate determination of the border of the tumor region is sometimes difficult (4). The detection and diagnosis of gastric tumor by image-enhanced endoscopy are still not good enough partly because the full utilization of the technique often needs magnification of the target region. For bladder cancer and glioblastoma, PDD has been widely used for the detection of the cancerous region $(5,6)$. The patient first takes 5-ALA orally from 2 to $4 \mathrm{~h}$ before the diagnosis. Then, the patient undergoes cystoscopic inspection for bladder cancer, or the tissue specimen is exposed by its fluorescence under a microscope for glioblastoma. The cancerous region is identified as a reddish area compared with the normal tissue. One potential problem of PDD is the difficulty in quantifying the intensity of the fluorescence (7), owing to the so-called photobleaching effect, which is a phenomenon where the fluorescent light intensity decreases under ultraviolet (UV) light exposure. The effectiveness of PDD with oral intake of 5-ALA in digestive disease is studied for intraoperative diagnosis of gallbladder cancer (8), peritoneal metastasis in colorectal cancer (9) and metastatic lymph node of gastric cancer (10), and so on. With regard to PDD for gastric tumors, it has been shown that this technique together with 5-ALA is useful for the early detection of the disease (11). However, one of the problems encountered is that the nontumorous mucosa also emits fluorescence in PDD. Thus, the fact that the fluorescence quickly diminishes upon exposure to UV light is problematic in PDD, since UV light inspection of the tumor region sometimes causes the fluorescence difference between the tumorous and nontumorous areas to be weak. For PDD using 5-ALA and UV light exposure, it is well known that the spectrum peaks at $630 \mathrm{~nm}$, which is the fluorescence peak of protoporphyrin IX (PPIX), the main metabolite of 5-ALA in the living cell.

The photobleaching effect of 5-ALA PDD or PPIX itself has been studied by several researchers, for rat and human esophagus (12), for mouse skin with topical application of 5-ALA (13), and for PPIX itself (14). They found that the primary peak at around $630 \mathrm{~nm}$ of the fluorescence of the PPIX decays quickly as exposed with light, while secondary broad peak with maximum at around $670 \mathrm{~nm}$ arises. One research group deduced that the secondary peak originates from the photoproducts of PPIX, possibly porphyrinchlorin dimer (14). The photobleaching effect has not yet been studied for 5-ALA PDD for human gastric sample, in which the photobleaching effect can have significant effect on the objectivity of 5-ALA PDD. In this regard, the normal gastric mucosa emits fluorescence in 5-ALA PDD. We have studied the photobleaching effect in 5-ALA PDD for human gastric samples in detail, to propose the method for possibly minimizing the photobleaching effect of the 5-ALA PDD.

\section{Methods}

\section{New endoscopic system for PDD}

The new endoscopic system $\left(\mathrm{Sie}-\mathrm{P} 2^{\circledR}\right)$ for PDD of the upper gastrointestinal tract was developed by Fujifilm Corporation (Tokyo, Japan). The endoscope has white light, NBI, and PDD inspection modes. With the newly equipped PDD mode, UV light of $410 \mathrm{~nm}$ wavelength is emitted from the distal end of the endoscopy. The source of the UV light is 

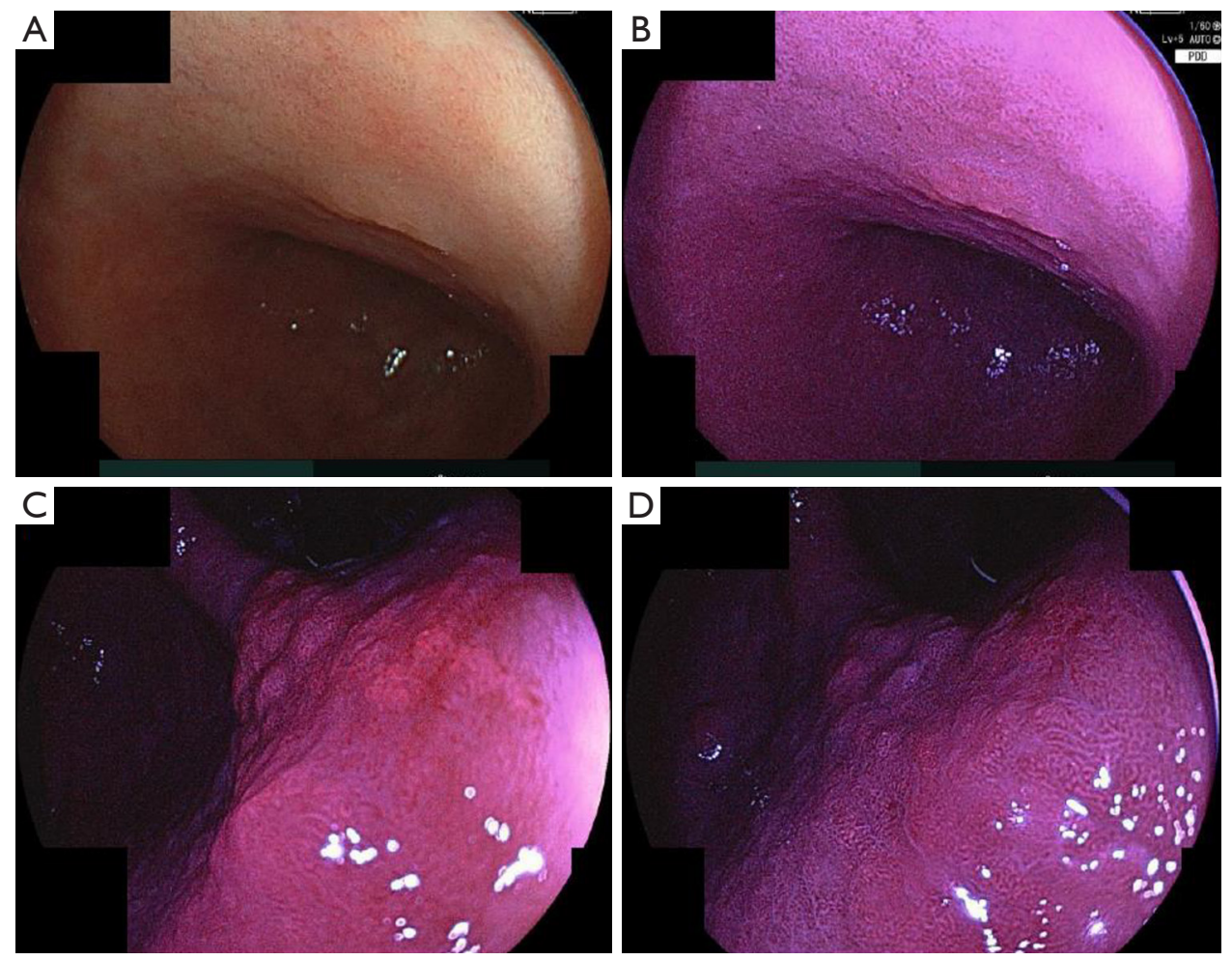

Figure 1 Pictures of (A) white light (B) PDD endoscopy of the II-a region(adenocarcinoma) in the lesser curvature of the lower body of the stomach. Pictures of the PDD endoscopy of II-a region(adenocarcinoma) in the lesser curvature of the angle of the stomach, showing photobleaching effect: (C) initial view at the start of the PDD endoscopy, (D) after observing about 2 minutes inspection of the PDD endoscopy.

a laser, which has a higher emission intensity than that of the previous system $\left(\right.$ Sie- $\left.P 1^{\circledR}\right)$. The fluorescence is detected with the complementary metal oxide semiconductor (CMOS) detector of the endoscope. Upon exposure to UV light, the tumorous region is expected to be visualized as a brighter spot compared with the non-tumorous region, reflecting the fact that the tumor is expected to emit more fluorescent light.

Example case of PDD endoscopy of gastric tumor with $\mathrm{Si} 2-\mathrm{P} 2^{\circledR}$ system is reported in the literature (15). Example cases of PDD using Si2 $\mathrm{P} 2{ }^{\circledR}$ system are presented in Figure 1. The region spotted as tumor before the PDD endoscopy is the 0 -IIa region (adenoma) in the lesser curvature of the lower body of the stomach. The picture of the white light endoscopy of the region is presented in Figure 1A. The picture of the fluorescent light endoscopy is presented in Figure $1 B$, in which the diagnosed tumor region is depicted as reddish region in the fluorescent light endoscopy. Figure $1 C$ shows picture of the PDD endoscopy of II-a region(adenocarcinoma) in the lesser curvature of the angle of the stomach. Figure $1 D$ shows the picture after about
2 minutes inspection of the PDD endoscopy, the brightness of the region becomes weak such that the contrast between tumor region and non-tumor region becomes weak.

\section{Two-dimensional spectrometer using a liquid crystal tunable filter and CMOS detector}

In this study, the fluorescence spectra of gastric tumor and non-tumorous samples in PDD with oral intake of 5-ALA was investigated using a two-dimensional (2D) spectrometer, which is newly developed by Murata Corporation, Kyoto, Japan, that utilizes a liquid crystal tunable filter (LCTF). The spectrometer is schematically depicted in Figure 2. In this system, the sample examined is exposed to a light source, which can be either white light or UV light. The light emitted from the sample is detected with a CMOS detector through the tunable bandpass filter whose center frequency is controlled by the voltage applied. The root mean square of the resolution of the bandpass filter is in the order of several nanometers. The range of the detected wavelength is from 500 to $800 \mathrm{~nm}$. Hereinafter, 


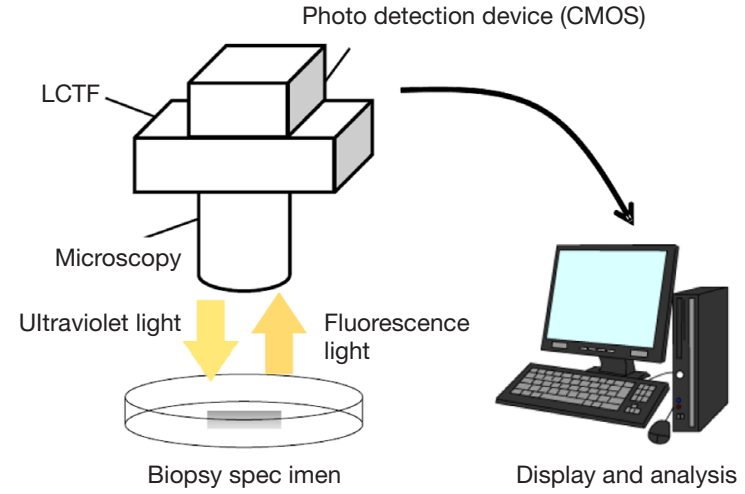

Figure 2 Schematic view of the 2-dimentional spectrometer using LCTF (developed by Murata Corporation, Kyoto, Japan).

the system is referred to as the LCTF system.

\section{Application of the developed photodynamic diagnostic endoscope and spectrometer to gastric tumor cases}

We investigated six cases of gastric tumors (early gastric cancer or adenoma) that were to be resected by endoscopic submucosal dissection (ESD). The region to be resected was diagnosed by biopsy as being tumorous (adenoma or cancer) before the PDD and ESD were performed. One case of gastric tumor presented as follows. The region spotted as being tumorous before the PDD was the 0 -IIa region in the lesser curvature of the lower body of the stomach. The white-light endoscopic image of the region is presented in Figure $2 A$ and the fluorescence endoscopic image is presented in Figure 2B. The diagnosed tumorous region was depicted as a reddish area by the fluorescence endoscopy.

Prior to the ESD, the upper gastrointestinal tract of the patient was first inspected using the newly developed photodynamic diagnostic endoscopic system. Then, the region diagnosed as the tumor (cancerous or adenomatous) was biopsied. The assumed non-tumorous region was also biopsied for comparison. Then, the biopsied specimens were inspected with the newly developed LCTF system to obtain the fluorescence spectrum of the specimen exposed to UV light. The center frequency of the LCTF was scanned from 580 to $715 \mathrm{~nm}$ at $5-\mathrm{nm}$ intervals, which took approximately $150 \mathrm{sec}$, and thus the exposure time to the UV light was $150 \mathrm{sec}$ per single measurement. Using this LCTF system, several consecutive measurements of each sample were taken for estimation of the photobleaching effect.

The biopsied specimens are pathologically diagnosed according to the group classification of biopsied specimen, described in the Japanese classification of gastric cancer, $3^{\text {rd }}$ English edition (16). In this classification, the group 1 sample denotes non-tumor, group 3 sample denotes adenoma and group 5 sample denotes cancer.

We also investigated the intrinsic spectrum of PPIX using the LCTF system. The PPIX was purchased from Frontier Scientific, Inc., (Logan, UT, USA) and dissolved in saline to a concentration of $0.01 \mu \mathrm{g} / \mathrm{mL}$.

\section{Calculation of the spectral data obtained by the liquid crystal tunable filter system}

The raw data from the CMOS detector of the LCTF system consisted of 772 pixels for the $\mathrm{x}$-axis and 580 pixels for the $y$-axis. First, to avoid instability, the values of four points [viz., $r(x, y), r(x+1, y), r(x, y+1)$, and $r(x+1, y+1)$ ] were averaged, resulting in 193 points for the $x$-axis and 145 points for the $\mathrm{y}$-axis [denoted as $\mathrm{R}(\mathrm{X}, \mathrm{Y})$ ]. The value of the raw measurement $\mathrm{R}(\mathrm{X}, \mathrm{Y}, \mathrm{f})$ was then subtracted by the background [viz., $\mathrm{B}(\mathrm{f})$, where $\mathrm{f}$ is the center tuning frequency of the LCTF], which is assumed to be constant in the CMOS plane.

$B(f)$ was calculated as the average of the four corner points of the CMOS plane (Figure $S 1$ shows examples of the raw measurements $\mathrm{R}(\mathrm{X}, \mathrm{Y}, \mathrm{f})$ for fixed $\mathrm{x}$ and $\mathrm{y}$, the background values $\mathrm{B}(\mathrm{f})$, and the background-subtracted values $\mathrm{Rsub}(\mathrm{X}, \mathrm{Y}, \mathrm{f})$ [i.e., $\mathrm{R}(\mathrm{X}, \mathrm{Y}, \mathrm{f})-\mathrm{B}(\mathrm{f})$ ], over the spectral range measured. The fluorescence spectrum had a primarily peak at $630 \mathrm{~nm}$ and a second broad peak from 660 to $700 \mathrm{~nm}$.

\section{Results}

\section{Evaluation of the photobleaching effect in biopsied tissue samples}

Figure $3 A$ shows the typical change of the consecutive measurements of the spectrum for the group 5 sample, for the same point in the 2 -dimentional plane. Figure $3 B$ shows the average ratio of the altitude of the specified wavelength, at the second to fourth measurement relative to that of the first measurement. The ratio is averaged over points in one sample. It is shown that the primary peak of the fluorescent light at $630 \mathrm{~nm}$ decreases quickly by the exposure on the UV light of the consecutive measurements. The average altitude of the spectrum from 660 to $700 \mathrm{~nm}$ also decreases by the exposure, but the degree of the decrease is not so prominent 

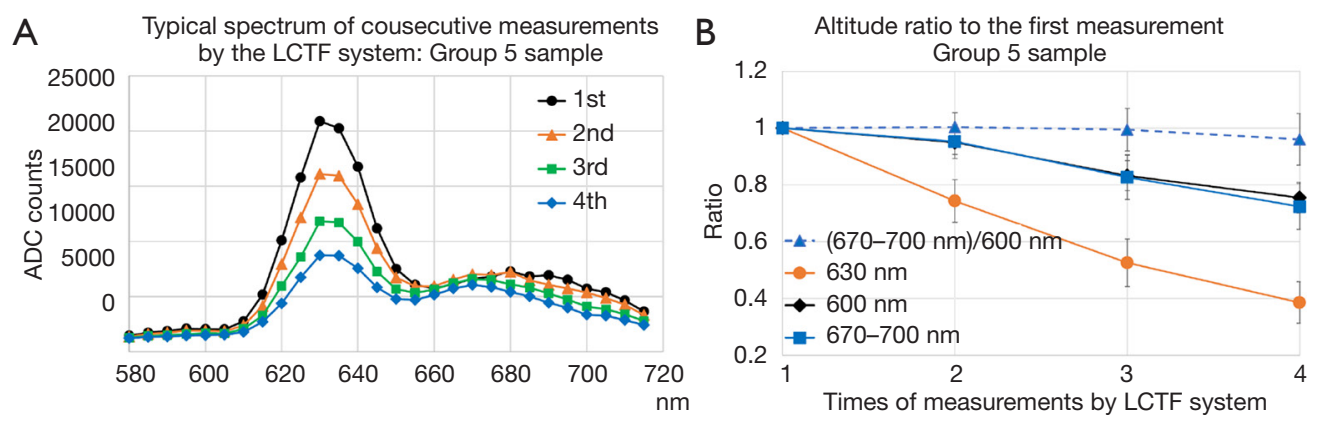

Figure 3 Spectrums and their properties in consecutive measurements by the LCTF system. (A) Typical spectrum of group 5 sample measured consecutively by the LCTF system: first measurement (circle), second measurement (triangle), third measurement (square), fourth measurement (diamond); (B) average altitude ratio of each (first to fourth) measurement relative to the first measurement for the average of 660-700 nm (square), $600 \mathrm{~nm}$ (diamond), $630 \mathrm{~nm}$ (circle), the average of 660-700 nm normalized by $600 \mathrm{~nm}$ (triangle), for group 5 sample. Error bar shows root mean square of the distribution.
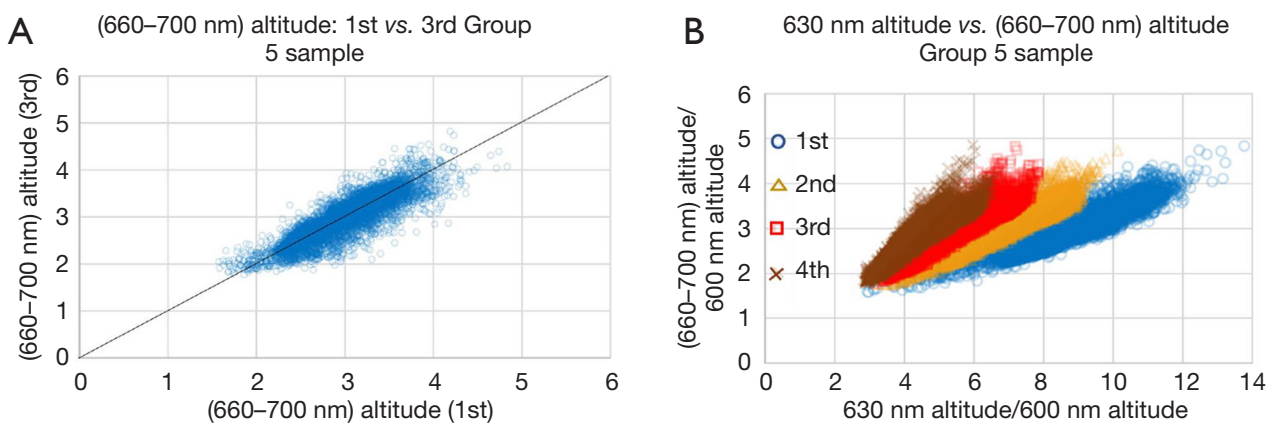

Figure 4 Properties of $(660-700 \mathrm{~nm})$ altitude in consecutive measurements by the LCTF system. (A) Correlation between first (x-axis) vs. third (y-axis) measurement for the average altitude from 660 to $700 \mathrm{~nm}$, normalized by $600 \mathrm{~nm}$ altitude. Each point corresponds to point in $\mathrm{x}-\mathrm{y}$ plane, for group 5 sample; (B) correlation between altitude of $630 \mathrm{~nm}$ normalized by altitude of $600 \mathrm{~nm}$ (x-axis) vs. average altitude from 660 to $700 \mathrm{~nm}$ normalized by altitude of $600 \mathrm{~nm}$ (y-axis), for each consecutive measurement of first (circle), second (triangle), third (square) and fourth (cross) for group 5 sample.

compared to the decrease of the altitude at $630 \mathrm{~nm}$. Additionally, the average altitude of the spectrum from 660 to $700 \mathrm{~nm}$ normalized by the altitude of $600 \mathrm{~nm}$, is almost constant up-to third measurement.

Figure $4 A$ shows the scattered plot of the average altitude of the spectrum from 660 to $700 \mathrm{~nm}$ of the first measurement $v s$. that of the third measurement, for group 5 sample. Each scattered point corresponds to each point at the $x-y$ plane of the CMOS, with requirement that the $630 \mathrm{~nm}$ altitude be more than 2.2 times higher than the altitude at $600 \mathrm{~nm}$. The correlation between the altitude of the first measurement and the third measurement is good.

Figure $4 B$ shows the scattered plot of the average altitude of the spectrum from 660 to $700 \mathrm{~nm} v s$. the altitude of
$630 \mathrm{~nm}$ normalized by the altitude of $600 \mathrm{~nm}$, for each measurement, for group 5 sample. Each scattered point corresponds to each point at the $x-y$ plane of the CMOS, with the same requirement that the $630 \mathrm{~nm}$ altitude be more than 2.2 times higher than the altitude at $600 \mathrm{~nm}$. The correlation between the average altitude of the spectrum from 660 to $700 \mathrm{~nm}$ and the altitude of $630 \mathrm{~nm}$ is good for each point, in each measurement.

Figure $5 A, B$ show the ratio of the altitude of the specified wavelength, at the second to third measurement relative to that of the first measurement, for three group 5 samples (Figure 5A), for three samples group 1 samples (Figure 5B). Average altitude from 660 to $700 \mathrm{~nm}$ normalized to that of the $600 \mathrm{~nm}$ is relatively constant from first to third 

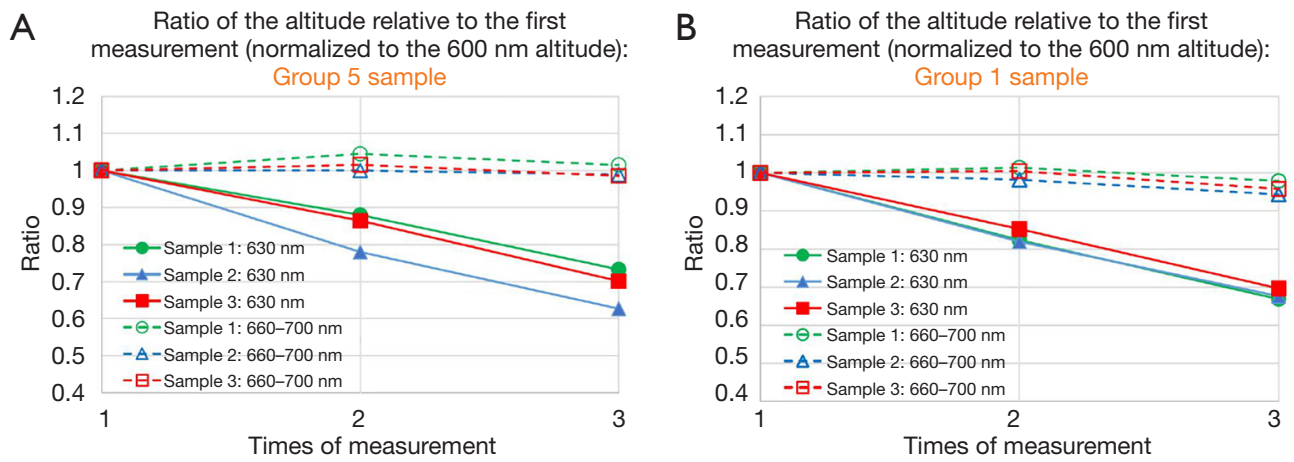

Figure 5 Average altitude ratio of each (first to three) measurement relative to the first measurement, for $630 \mathrm{~nm}$ altitude (solid), and average altitude from 660 to $700 \mathrm{~nm}$ (open) for three group 5 samples (A) and for three group 1 samples (B).

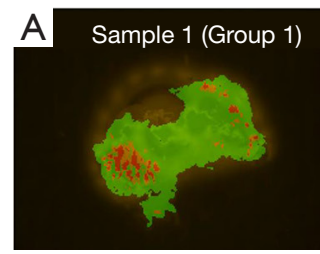

Sample 3 (Group 5)

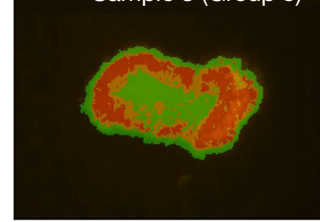

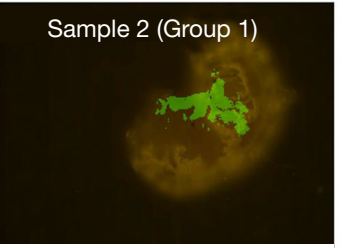

Sample 4 (Group 5)

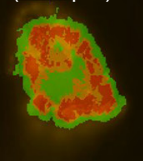

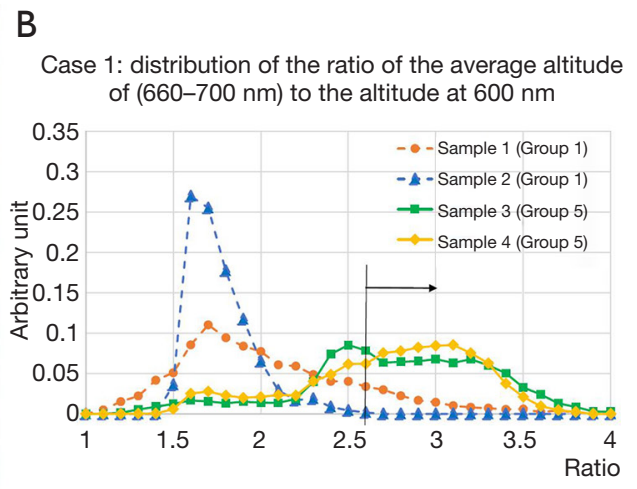

Figure 6 Comparison of (660-700 nm) altitude between group 1 and group 5 samples. (A) Two-dimensional plot for the two group 1 samples and two group 5 samples of case 1. Each point is attributed to corresponding color according to the ratio of average altitude from 660 to $700 \mathrm{~nm}$ to the altitude of $600 \mathrm{~nm}$ : red: $3.0<(660-700 \mathrm{~nm}) / 600 \mathrm{~nm}$, orange: $2.6<(660-700 \mathrm{~nm}) / 600 \mathrm{~nm}<3.0$, green: $(660-700$ $\mathrm{nm}) / 600 \mathrm{~nm}<2.6$. The plots are overlaid by the white light picture of the samples; (B) distribution of the average altitude from 660 to 700 $\mathrm{nm}$ normalized to the altitude of $600 \mathrm{~nm}$, for two group 1 samples and two group 5 samples.

measurement, within 5\% deviation, compared to the fact that the altitude of $630 \mathrm{~nm}$ normalized to that of the $600 \mathrm{~nm}$ decreases as the sample is exposed to ultraviolet light. The above statement is also valid for group 3 samples (Figure S2). In summary, we see similar property of the photobleaching effect described above, for group 1, group 3 and group 5 samples.

\section{Case examples}

Next, we compare the average altitude from 660 to 700 $\mathrm{nm}$ normalized to the altitude of $600 \mathrm{~nm}$, which is well correlated to the altitude of $630 \mathrm{~nm}$, between group 5 sample and group 1 sample for the case 1 , and between group 3 and group 1 sample for the other case 2 .

\section{Case example 1: a 72-year-old male}

Figure $6 A$ show the 2-dimentional plot for two group 1 samples, for two group 5 samples, with the condition that altitude at $630 \mathrm{~nm}$ is higher than 2.2 times of altitude at $600 \mathrm{~nm}$. The requirement eliminates the background, effectively selecting tissue. Each point is attributed to corresponding color according to the ratio of average altitude from 660 to $700 \mathrm{~nm}$ normalized to the altitude of $600 \mathrm{~nm}$ : red: $3.0<(660-700 \mathrm{~nm}) / 600 \mathrm{~nm}$, orange: $2.6<$ $(660-700 \mathrm{~nm}) / 600 \mathrm{~nm}<3.0$, green: $(660-700 \mathrm{~nm}) / 600 \mathrm{~nm}$ $<2.6$.

Figure $6 B$ shows the distribution of the average altitude from 660 to $700 \mathrm{~nm}$ normalized to the altitude of $600 \mathrm{~nm}$, for same samples with the same requirement as in Figure $6 \mathrm{~A}$. Distributions of the ratio for group 5 samples tend to 

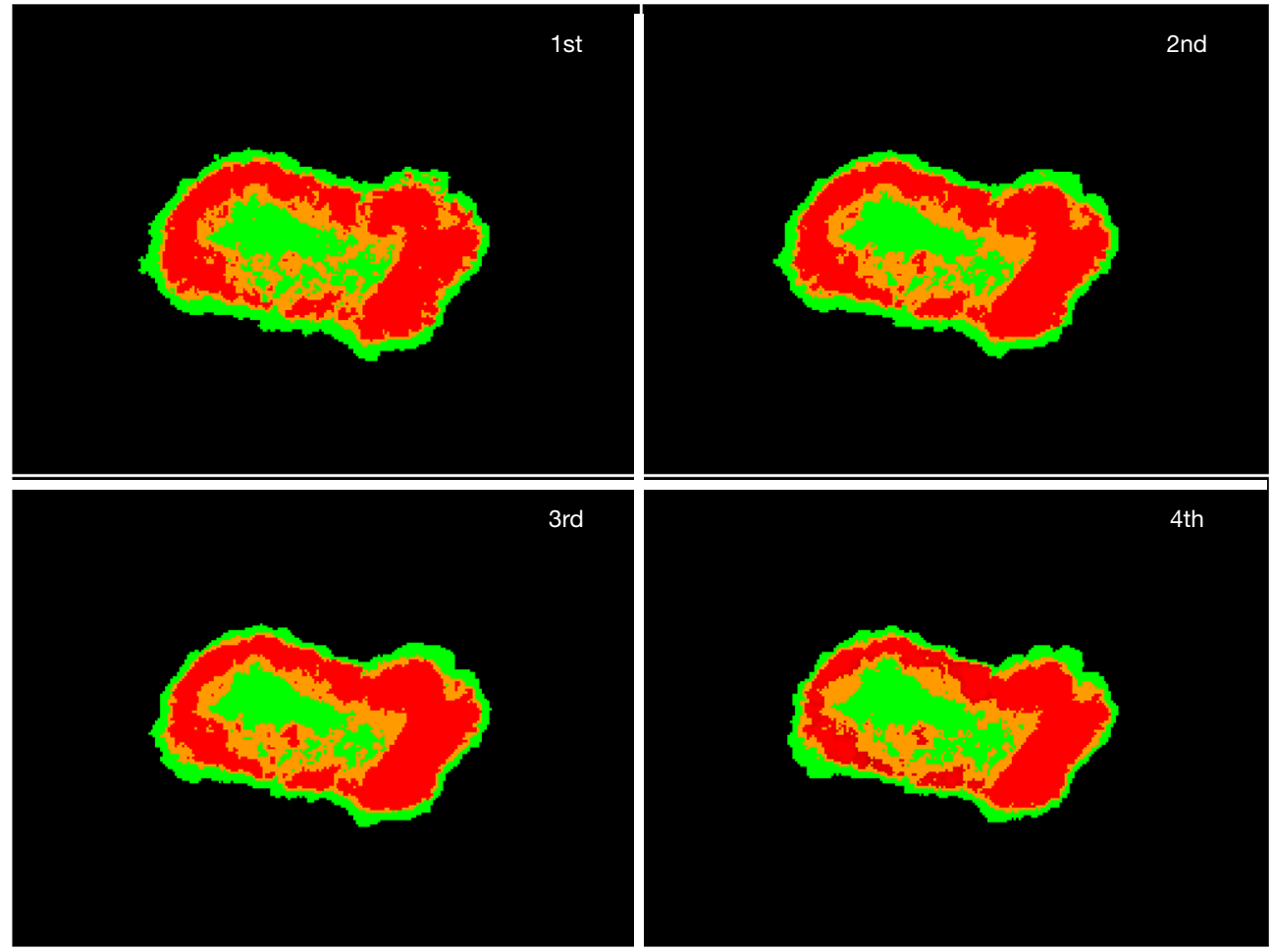

Figure 7 Two-dimensional plot for the group 5 sample (same sample as Figure $6 \mathrm{~A}$ ) for four consecutive measurements (1st, 2nd, 3rd, 4th). Each point is attributed to corresponding color according to the ratio of average altitude from 660 to $700 \mathrm{~nm}$ to the altitude of $600 \mathrm{~nm}$ : red: $3.0<(660-700 \mathrm{~nm}) / 600 \mathrm{~nm}$, orange: $2.6<(660-700 \mathrm{~nm}) / 600 \mathrm{~nm}<3.0$, green: $(660-700 \mathrm{~nm}) / 600 \mathrm{~nm}<2.6$.

have higher value compared to group 1 samples. It can be estimated that the group 5 samples have higher altitude of $630 \mathrm{~nm}$, in the same condition of photo-bleaching, since the average altitude from 660 to $700 \mathrm{~nm}$ has good correlation to the altitude of $630 \mathrm{~nm}$.

Figure 7 shows the same plot as Figure 6A, with the consecutive 4 times measurements by the system, in which the point has color in the same way as in Figure 6A. The plots of the consecutive measurements have similar view between measurements, which reflects the fact that the photobleaching effect is small by using the average altitude from 660 to $700 \mathrm{~nm}$ normalized to the altitude of $600 \mathrm{~nm}$.

\section{Case example 2: a 60-year-old male}

Figure $8 A$ show the 2-dimentional plot for two group 1 samples, for two group 3 samples, with the condition that altitude at $630 \mathrm{~nm}$ is higher than 2.2 times of altitude at $600 \mathrm{~nm}$. Each point is attributed to corresponding color in the same way as in Figure 6A. Figure $8 B$ shows the distribution of the average altitude from 660 to $700 \mathrm{~nm}$ normalized to the altitude of $600 \mathrm{~nm}$, for same samples with the same requirement as in Figure 8A. Distributions of the ratio for group 3 samples tend to have higher tail compared to group 1 samples.

Figure 9 shows the HE-stained sample. Adenoma ductal cells can be spotted. By comparing HE stained sample (Figure $9 B$ ) and 2-dimentional plot of the ratio of the altitude (Figure 9A), it can be stated that region where the adenoma ductal cells are dominant has higher average altitude from 660 to $700 \mathrm{~nm}$ normalized to the altitude of $600 \mathrm{~nm}$, compared to the non-tumor region. The plots of the consecutive measurements have similar view between measurements, especially up to third measurement, as in case 1 (Figure S3), which shows that the photobleaching effect is small by using the average altitude from 660 to $700 \mathrm{~nm}$ normalized to the altitude of $600 \mathrm{~nm}$.

\section{Discussion}

In this study, it is shown that the average altitude between 660 and $700 \mathrm{~nm}$ is not so much influenced by the photobleaching effect as the $630 \mathrm{~nm}$ primary peak altitude, 


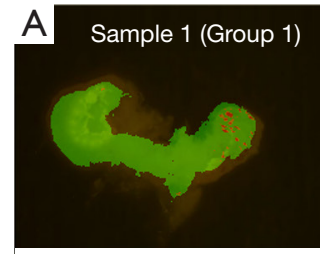

Sample 3 (Group 3)

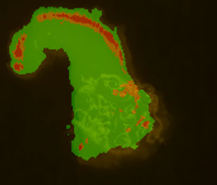

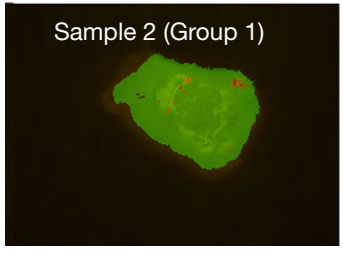

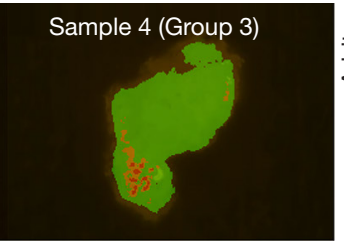

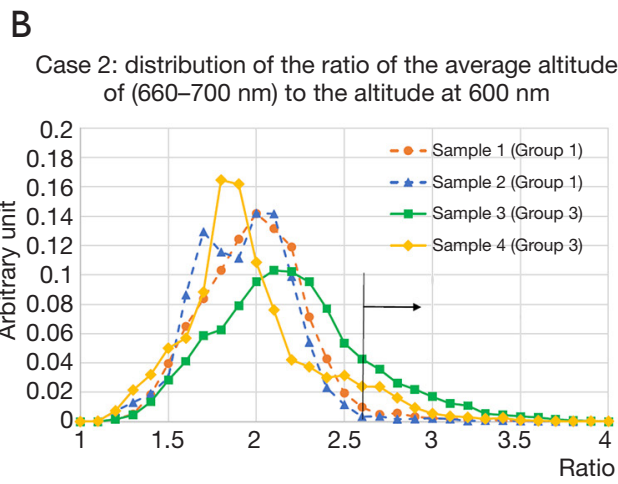

Figure 8 Comparison of (660-700 nm) altitude between group 1 and group 3 samples. (A) Two-dimensional plot for the two group 1 samples and two group 3 samples of case 2. Each point is attributed to corresponding color according to the ratio of average altitude from 660 to $700 \mathrm{~nm}$ to the altitude of $600 \mathrm{~nm}$ : red: $3.0<(660-700 \mathrm{~nm}) / 600 \mathrm{~nm}$, orange: $2.6<(660-700 \mathrm{~nm}) / 600 \mathrm{~nm}<3.0$, green: $(660-700$ $\mathrm{nm}) / 600 \mathrm{~nm}<2.6$. The plots are overlaid by the white light picture of the samples; (B) distribution of the average altitude from 660 to 700 $\mathrm{nm}$ normalized to the altitude of $600 \mathrm{~nm}$, for two group 1 samples and two group 3 samples.

A

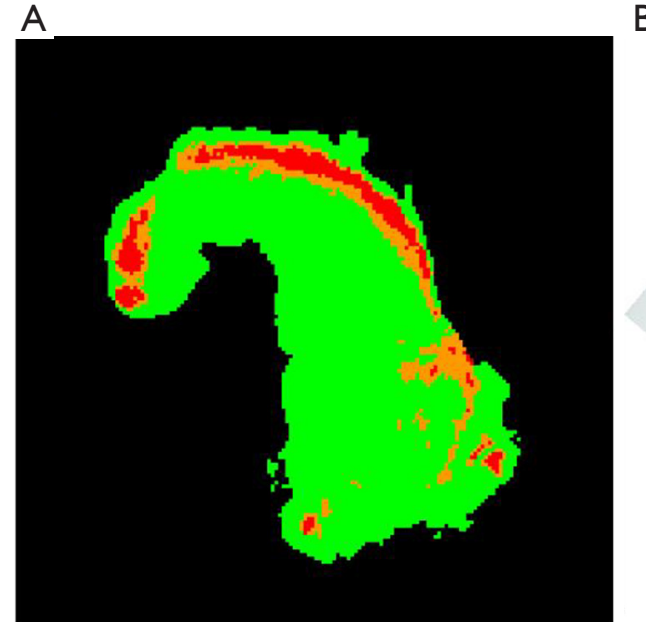

B

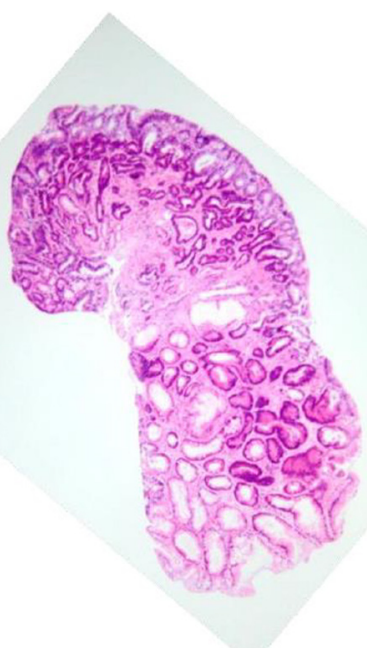

Figure 9 Comparison of $(660-700 \mathrm{~nm}$ ) altitude to histology. (A) Two-dimensional plot for the group 3 sample (same sample as Figure 8A). Each point is attributed to corresponding color according to the ratio of average altitude from 660 to $700 \mathrm{~nm}$ to the altitude of $600 \mathrm{~nm}$ : red: $3.0<(660-700 \mathrm{~nm}) / 600 \mathrm{~nm}$, orange: $2.6<(660-700 \mathrm{~nm}) / 600 \mathrm{~nm}<3.0$, green: $(660-700 \mathrm{~nm}) / 600 \mathrm{~nm}<2.6$; (B) HE staining of the sample.

which is the property observed irrelevantly whether the tissue is tumor (adenoma or adenocarcinoma) or nontumor. Additionally, the $630 \mathrm{~nm}$ altitude and altitude from 660 to $700 \mathrm{~nm}$ of the fluorescent light in 5-ALA PDD correlates well. Therefore, altitude between 660 and $700 \mathrm{~nm}$ can be utilized in 5-ALA PDD such that there is less photobleaching effect, thus eligible for more reliable and efficient identification of the tumor region in PDD. One possible procedure is applying the correction method emphasizing the spectrum between 660 and $700 \mathrm{~nm}$. The average altitude from 660 to $700 \mathrm{~nm}$ could also be used to define the value which reflects initial $630 \mathrm{~nm}$ peak altitude before photobleaching effect, which leads to quantification of PDD, opening new possibility of PDD using 5-ALA.

Figure 10 shows the intrinsic PPIX spectrum measured by the LCTF system in the same way as described. The second broad peak around $660 \mathrm{~nm}$ does not change much as exposed by the fluorescent light, whereas the 


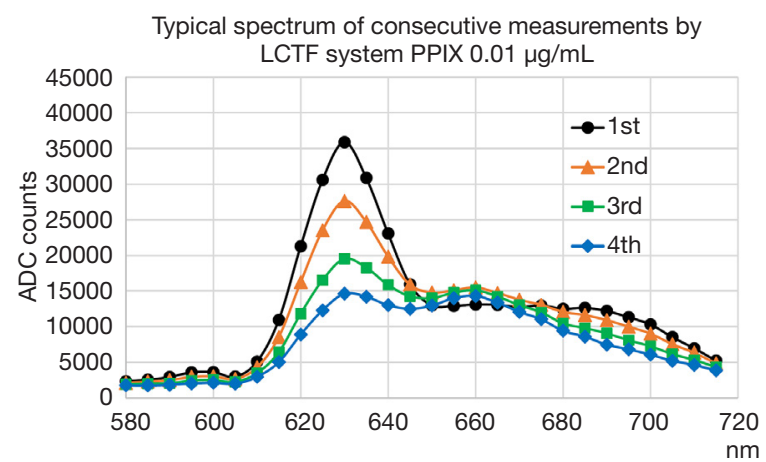

Figure 10 Typical spectrum of PPIX $(0.01 \mu \mathrm{g} / \mathrm{mL}$; diluted by saline) measured consecutively by the LCTF system: for first measurement (circle), second measurement (triangle), third measurement (square), fourth measurement (diamond).

primary $630 \mathrm{~nm}$ peak decreases quickly, which is similar behavior observed in the measurement of the tissue sample. Therefore, it is likely that the effect seen in the measurement of the tissue sample is the photobleaching effect of the PPIX itself. The observed effect is similar to that described in previous works from other groups (12-14), in which the second broad peak around $660 \mathrm{~nm}$ is possibly originated from the photoproducts of PPIX.

As it is shown in two cases of gastric tumor, there is the tendency that the tumor region has higher altitude from 660 to $700 \mathrm{~nm}$ than non-tumor region in same case. Since the $630 \mathrm{~nm}$ altitude of the fluorescent light and that from 660 to $700 \mathrm{~nm}$ in 5-ALA PDD correlates well, it could be argued that the original $630 \mathrm{~nm}$ altitude of the PPIX fluorescence is higher in tumor region than non-tumor region. The next thing to be explored is the comparison of the intensity of the PPIX fluorescence between tumor regions and non-tumor regions with larger samples to confirm this assumption, using the average altitude from 660 to $700 \mathrm{~nm}$.

\section{Conclusions}

In conclusion, we have ascertained in this study that the 5-ALA PDD using the average fluorescence altitude of 660$700 \mathrm{~nm}$ (normalized to the altitude at $600 \mathrm{~nm}$ ) instead of the peak altitude at $630 \mathrm{~nm}$, is considered to be more effective in distinguishing between tumorous and non-tumorous tissues, because of the lower photobleaching effect at this specific spectral range. Our findings are expected to greatly improve the objective diagnosis of gastrointestinal cancers by 5-ALA-based photodynamic diagnostic endoscopy.

\section{Acknowledgments}

Funding: We are very grateful for the collaboration with members of Murata corporation who participated in the joint research project. The research is performed under the funding of Grant-in-Aid for Scientific Research.

\section{Footnote}

Conflicts of Interest: HI serves as an unpaid editorial board member of Annals of Translational Medicine from Jan 2018 to Dec 2019. The other authors have no conflicts of interest to declare.

Ethical Statement: The authors are accountable for all aspects of the work in ensuring that questions related to the accuracy or integrity of any part of the work are appropriately investigated and resolved. This clinical research is approved by the Tottori University ethics committee and written informed consent was obtained from all patients.

Open Access Statement: This is an Open Access article distributed in accordance with the Creative Commons Attribution-NonCommercial-NoDerivs 4.0 International License (CC BY-NC-ND 4.0), which permits the noncommercial replication and distribution of the article with the strict proviso that no changes or edits are made and the original work is properly cited (including links to both the formal publication through the relevant DOI and the license). See: https://creativecommons.org/licenses/by-nc-nd/4.0/.

\section{References}

1. Nakayoshi T, Tajiri H, Matsuda K, et al. Magnifying endoscopy combined with narrow band imaging system for early gastric cancer: correlation of vascular pattern with histology (including video). Endoscopy 2004;36:1080-4.

2. Yagi k, Nakamura A, Sekine A, et al. Magnifying endoscopy combined with narrow band imaging for early differentiated gastric adenocarcinoma. Dig Endosc 2009;20:115-22.

3. Kaise M, Kato M, Urashima M, et al. Magnifying endoscopy with narrow-band imaging for differential diagnosis of superficial depressed gastric lesions. Endoscopy 2009;41:310-5. 
4. Yoshinaga S, Oda I, Abe S, et al. Evaluation of the margins of differentiated early gastric cancer by using conventional endoscopy. World J Gastrointest Endosc 2015;7:659-64.

5. Jocham D, Stepp H, Waidelich R, et al. Photodynamic diagnosis in urology: State-of-the-art. Eur Urol 2008;53:1138-48.

6. Kaneko S. Recent Advances in PDD and PDT for Malignant Brain Tumors. Available online: https:/www. jstage.jst.go.jp/article/lsj/36/APLS/36_APLS_1351/_pdf/char/en

7. Unresolved Problem in 5-aminolevulinic Acid Based Photodynamic Diagnosis Quantification of Fluorescence and Molecular Mechanism of Porphyrin Accumulation. Available online: https://www.jstage.jst.go.jp/article/ jslsm/32/2/32_2_143/_pdf/-char/en

8. Kamada Y, Murayama Y, Arita T, et al. Intraoperative 5-aminolevulinic acid-mediated photodynamic diagnosis of gallbladder cancer: A case report. Photodiagnosis Photodyn Ther 2016;14:74-6.

9. Kondo Y, Murayama Y, Konishi H, et al. Fluorescent detection of peritoneal metastasis in human colorectal cancer using 5-aminolevulinic acid. Int J Oncol 2014;45:41-6.

10. Koizumi N, Harada Y, Murayama Y, et al. Detection of metastatic lymph nodes using 5-aminolevulinic

Cite this article as: Yamashita T, Kinoshita H, Sakaguchi T, Isomoto H. Objective tumor distinction in 5-aminolevulinic acid-based endoscopic photodynamic diagnosis, using a spectrometer with a liquid crystal tunable filter. Ann Transl Med 2020;8(5):178. doi: 10.21037/atm.2020.01.108
Acid in patients with gastric cancer. Ann Surg Oncol 2013;20:3541-8.

11. Isomoto H, Ogihara K, Seno T, et al. 5-Aminolevulinic Acid mediated Photodynamic Diagnosis with A LaseEquipped Video Image Endoscope. Available online: https://www.jstage.jst.go.jp/article/jslsm/36/2/36_ jslsm-36_0016/_pdf/-char/en

12. Pogue BW, Sheng C, Benevides J, et al. Protoporphyrin IX fluorescence photobleaching increases with the use of fractionated irradiation in the esophagus. J Biomed Opt 2008;13:034009.

13. Juzenas P, Iani V, Bagdonas S, et al. Fluorescence spectroscopy of normal mouse skin exposed to 5-aminolaevulinic acid and red light. J Photochem Photobiol B 2001;61:78-86.

14. Bagdonas S, Ma LW, Iani V, et al. Phototransformations of 5-Aminolevulinic Acid-induced Protoporphyrin IX in vitro: A Spectroscopic Study. Photochem Photobiol 2000;72:186-92.

15. Sakaguchi T, Kinoshita H, Isomoto H, et al. Modified laser-based photodynamic endoscopic diagnosis for early gastric cancer. Dig Endosc 2019;31:e84-5.

16. Japanese Gastric Cancer Association. Japanese classification of gastric carcinoma: 3rd English edition. Gastric Cancer 2011;14:101-12. 


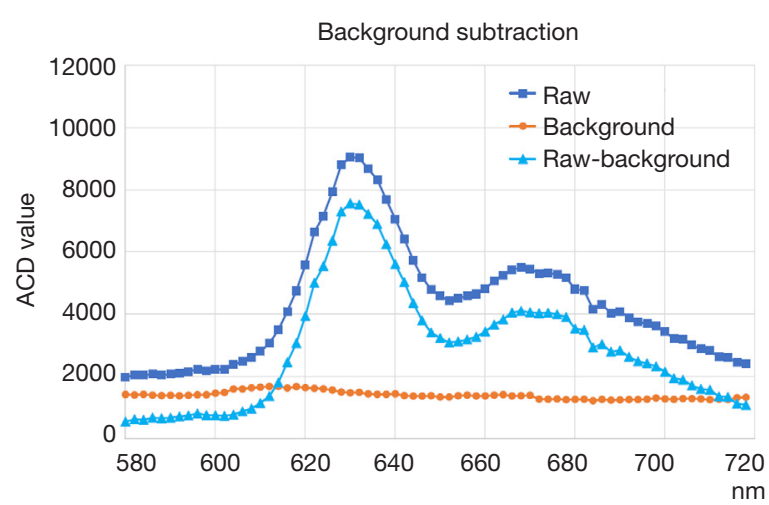

Figure S1 Measured spectrum before background subtraction $\mathrm{R}(\mathrm{X}, \mathrm{Y}, \mathrm{f})$ (square); calculated background spectrum $\mathrm{B}(\mathrm{f})$ (round); Subtracted spectrum Rsub(X,Y,f) (triangle).
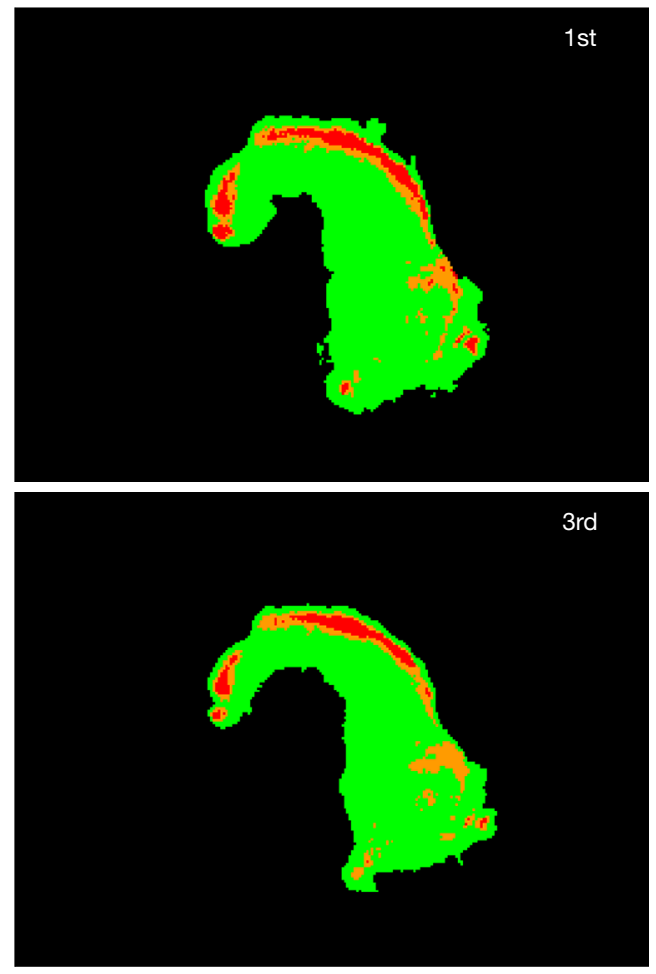

Ratio of the altitude relative to the first measurement (normalized to the $600 \mathrm{~nm}$ altitude)

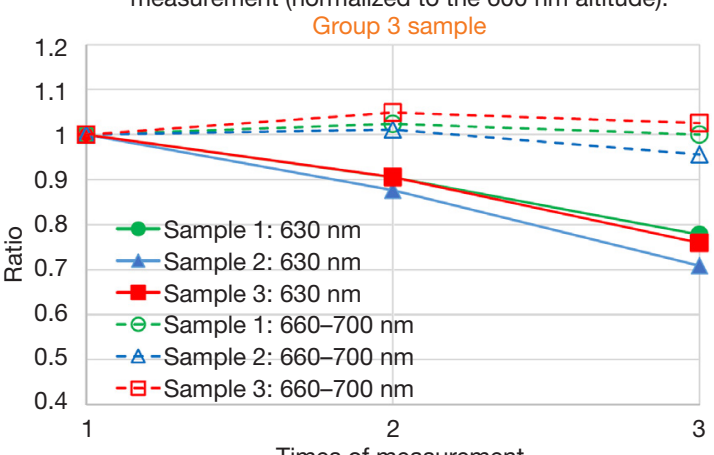

Times of measurement

Figure S2 Average altitude ratio of each (first to three) measurement relative to the first measurement, for $630 \mathrm{~nm}$ altitude (solid), and average altitude from 660 to $700 \mathrm{~nm}$ (open) for three group 3 samples.
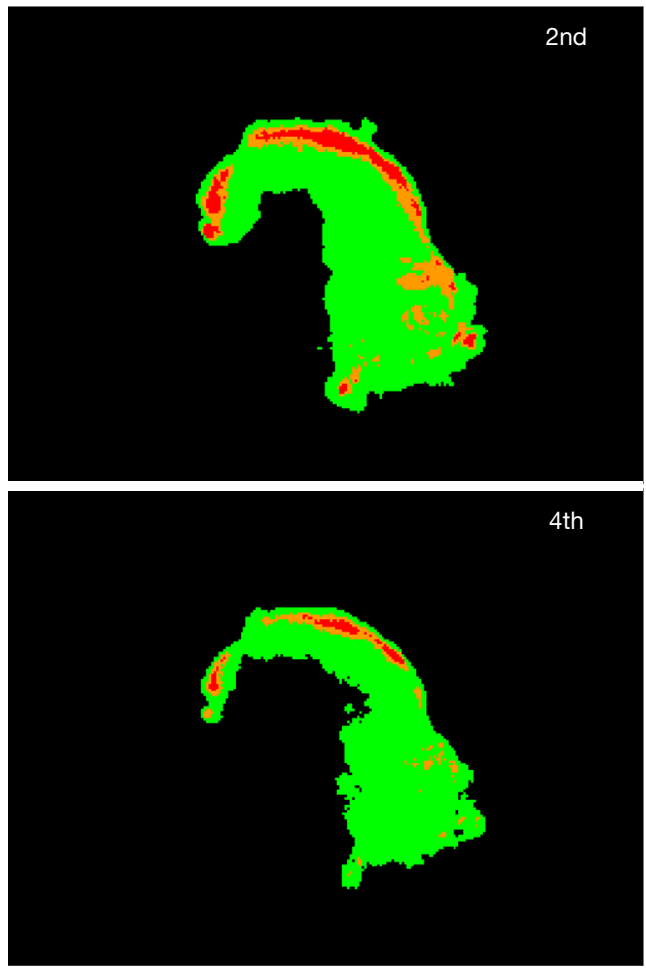

Figure S3 Two-dimensional plot for the group 3 sample (same sample as Figure 8A) for four consecutive measurements (1st, 2nd, 3rd, 4th). Each point is attributed to corresponding color according to the ratio of average altitude from 660 to $700 \mathrm{~nm}$ to the altitude of $600 \mathrm{~nm}$ : red: $3.0<(660-700 \mathrm{~nm}) / 600 \mathrm{~nm}$, orange: $2.6<(660-700 \mathrm{~nm}) / 600 \mathrm{~nm}<3.0$, green: $(660-700 \mathrm{~nm}) / 600 \mathrm{~nm}<2.6$. 